\title{
Oxygenation of saturated and unsaturated hydrocarbons with sodium periodate catalyzed by manganese(III) tetra-arylporphyrins, to study the axial ligation of imidazole
}

\author{
REZA TAYEBEE \\ Department of Chemistry, Sabzevar Teacher Training University, Sabzevar, Iran 397 \\ e-mail: rtayebee@sttu.ac.ir
}

MS received 4 June 2006; revised 8 July 2006

\begin{abstract}
Competitive oxygenation of cyclooctene and tetralin with sodium periodate catalyzed by $\mathrm{Mn}^{\text {(III)}}$ (TPP)OAc, TPP = meso-tetraphenylporphyrin; $\mathrm{Mn}^{\text {(III) }}$ (TNP)OAc, TNP = meso-tetrakis $(1$-naphthyl) porphyrin; $\mathrm{Mn}^{\text {(III) }}$ (TMP)OAc, TMP = meso-tetrakis(2,4,6-trimethyl-phenyl)porphyrin; $\mathrm{Mn}^{\text {(III) }}$ (TDCPP)OAc, TDCPP = meso-tetrakis(2,6-dichlorophenyl) porphyrin, and $\mathrm{Mn}^{\text {(III) }}\left(\mathrm{TPNMe}_{2}-\mathrm{TFPP}\right) \mathrm{OAc}, \mathrm{TPNMe}_{2^{-}}$ TFPP = meso-tetrakis(para-NMe $\mathrm{N}_{2}$-tetrafluorophenyl)porphyrin, was carried out in the presence or absence of imidazole. This study showed that, in the absence of imidazole, selectivity for epoxide formation was high with electron-rich catalysts such as Mn(TPP)OAc, Mn(TNP)OAc and Mn(TMP)OAc, but low with electron-deficient catalysts such as Mn(TDCPP)OAc and Mn(TPNMe 2 -TFPP)OAc. Presumably, not only the axial ligation of imidazole to the four-coordinate $\mathrm{Mn}(\mathrm{III})$-center, but also the steric and electronic influences of aryl-substituents on the porphyrin periphery affect the selectivity of the catalytic oxidation reaction.
\end{abstract}

Keywords. Imidazole; periodate; manganese(III); axial ligation; oxygenation of hydrocarbons; tetraarylporphyrins.

\section{Introduction}

Proximal influences of imidazoles as the fifth ligand coordinated to the iron(II) protoporphyrin IX of oxygen carrying hemoproteins such as hemoglobin and myoglobin affect the affinity of heme complexes for oxygen and carbon monoxide molecules. ${ }^{1-3}$ Such findings prompted the interest of several groups to obtain deeper insights into the role of nitrogen bases such as pyridine and imidazole on the oxygenation of hydrocarbons catalyzed by artificial metalloporphyrin model compounds, which mimic the enzymatic role of cytochrome P-450 and oxygen carrying hemoproteins. $^{4-10}$

Very few catalytic oxygenation protocols have discussed the activating role of axial ligands, such as imidazoles and pyridines, on the oxidation of hydrocarbons catalyzed by metalloporphyrins. ${ }^{11,12}$ Almost all of these studies have shown that the efficacy of oxygenation systems increases in the presence of a good nitrogen donor acting as a Lewis base. Our previous findings on the epoxidation of various olefins with sodium periodate have demonstrated that the catalytic system is capable of fast epoxidation in the absence of axial ligand. ${ }^{13,14}$ Presence of a catalytic amounts of a strong nitrogen donor, such as imidazole, has a strikingly large effect on the efficiency and selectivity of oxygenation of saturated and unsaturated hydrocarbons catalyzed by manganese(III) or iron(III) tetraarylporphyrins. ${ }^{14-17}$ These findings led us to investigate new aspects of the catalytic role of imidazole on the efficiency and product selectivity of $\mathrm{Mn}^{\text {(III) }}$ (Por)- $\mathrm{NaIO}_{4}$ oxygenation system. Scheme 1 illustrates the overall catalytic oxidation of hydrocarbons with sodium periodate-manganese(III) tetraarylporphyrin oxygenation system.

\section{Materials and methods}

Solvents, reagents, and other chemicals used in this study were of the highest grade available and were purchased from SD Fine Chemicals (India). The reagents were stored at $5^{\circ} \mathrm{C}$ and purified just before use. Silica gel 60 (70-230 mesh, purchased from E Merck A.G., Darmstadt, West Germany) was used for column chromatography. Porphyrin ligands were prepared by the modified method of Lindsey through 
condensation of pyrrole and the corresponding aldehydes. ${ }^{18}$ Manganese porphyrin complexes were prepared using the general procedure of Adler and were purified by column chromatography on neutral alumina eluted with $\mathrm{CHCl}_{3}{ }^{19}$ GLC analyses were performed on a Shimadzu-17A gas chromatograph using a flame ionization detector and equipped with $3 \mathrm{~m} \times 3 \mathrm{~mm}$ glass columns packed with $10 \%$ SE-30 on Chromosorb PAW (80-100 mesh) and fused silica WCOT $25 \mathrm{~m} \times 0.23 \mathrm{~mm}$ capillary column.

\subsection{General procedure for the oxygenation reaction}

All reactions were carried out at room temperature in air. In a typical reaction, cyclooctene $(0.5 \mathrm{mmol})$, tetralin $(1.5 \mathrm{mmol})$ and dichloromethane $(2 \mathrm{ml})$ were successively added to porphyrin catalyst $(0.006 \mathrm{mmol})$ in a round-bottomed flask $(25 \mathrm{ml})$. Then imidazole (if present, $0.06 \mathrm{mmol}$ ), tetrabutylammonium bromide $(0.06 \mathrm{mmol})$ and $\mathrm{NaIO}_{4}(1 \mathrm{mmol})$ in water $(10 \mathrm{ml})$ were added to this solution and the reaction mixture was stirred magnetically for the required time at $24 \pm 2{ }^{\circ} \mathrm{C}$. Progress of the reaction was followed by analysis via gas chromatography of aliquots withdrawn periodically from the reaction mixture after $0.5 \mathrm{~h}(8 \mathrm{~h})$. Products were identified by comparison of their GLC retention times with those of authentic samples. After completion of the reaction, products were extracted with dichloromethane. The extract was dried over anhydrous sodium sulfate, filtered and was concentrated under reduced pressure. The concentrated filtrate treated with 2,4-dinitrophenylhydrazine in $6 \% \mathrm{HCl}$ to give 2,4-dinitro-

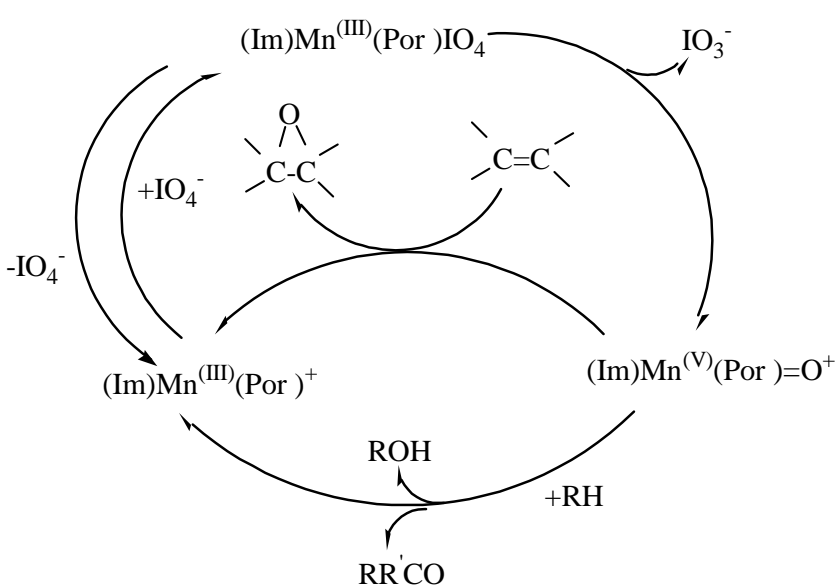

Scheme 1. Overall catalytic oxidation of hydrocarbons with (Im) $\mathrm{Mn}^{(\mathrm{III})}$ (Por)-NaIO 4 oxygenation system. phenylhydrazone of $\alpha$-tetralone. Then cyclooctene oxide was resolved from the organic phase and was purified by column chromatography on a silica gel pad eluted with $1: 10$ ethyl acetate : hexane.

\section{Results and discussion}

Several studies have described product selectivity displayed in the oxidation of a mixture of saturated and unsaturated hydrocarbons catalyzed by various metal tetraarylporphyrins. In contrast to many similar oxygenation protocols, ${ }^{11,12}$ the sodium periodatemanganese(III) porphyrin oxygenation system is a potent oxygenation method in the absence of axial ligand. ${ }^{14,15,20,21}$ These differences promoted our interest to investigate effects of imidazole on the competitive oxidation of tetralin, as a saturated cycloalkane, and cyclooctene, as an unsaturated cyclic olefin.

Table 1 summarizes the results of synchronous oxygenation of tetralin and cyclooctene with sodium periodate in the presence of imidazole catalyzed by different manganese(III) tetra-arylporphyrins (scheme 2). It has been found that oxygenation of olefins and aryl-alkanes led to the formation of epoxides and ketones respectively, as the major products in this catalytic oxidation system. ${ }^{12}$ Selectivity of cyclooctene epoxidation and oxygenation of tetralin to $\alpha$-tetralone exceeded $95 \%$ for all the manganese catalysts. Obviously, steric and electronic characteristics of the periphery of Mn-porphyrin catalysts affected
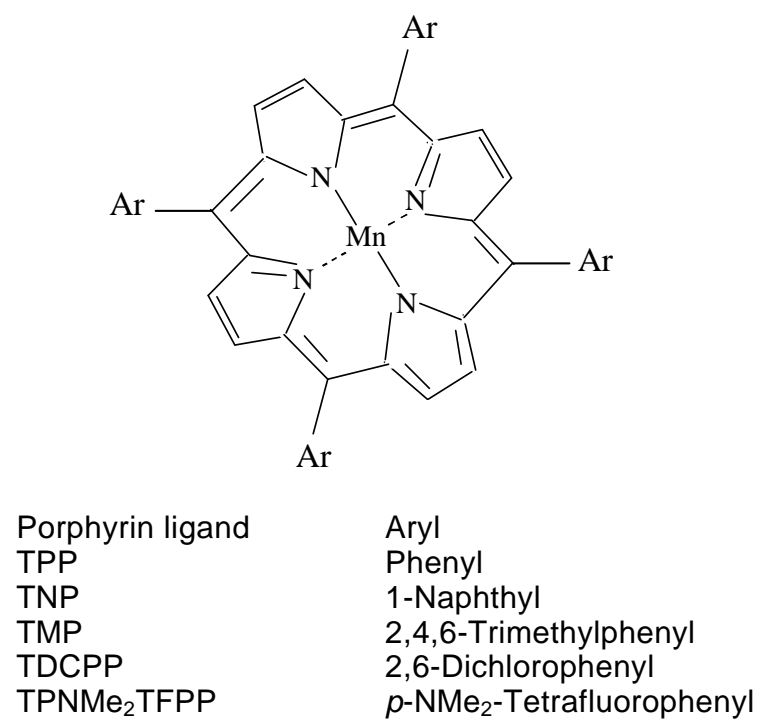

Scheme 2. General structure and abbreviations used for manganese porphyrins. 
Table 1. Competitive oxygenation of tetralin and cyclooctene with sodium periodate catalyzed by different manganese porphyrins in the presence of imidazole. ${ }^{\mathrm{a}}$

\begin{tabular}{|c|c|c|c|}
\hline Catalyst & Yield $\%$ of $\alpha$-tetralone $(\mathrm{A})^{\mathrm{b}}$ & Yield $\%$ of cyclooctene oxide $(B)^{b}$ & $(\mathrm{~B}) /(\mathrm{A})^{\mathrm{c}}$ \\
\hline $\mathrm{Mn}^{(\mathrm{III})}(\mathrm{TPP}) \mathrm{OAc}$ & $5 \cdot 1(19)$ & $13 \cdot 5(71)$ & $2 \cdot 6(3 \cdot 7)$ \\
\hline $\mathrm{Mn}^{(\mathrm{III})}(\mathrm{TNP}) \mathrm{OAc}$ & $6(19)$ & $19 \cdot 5(79)$ & $3 \cdot 2(4 \cdot 1)$ \\
\hline $\mathrm{Mn}^{(\mathrm{III})}(\mathrm{TMP}) \mathrm{OAc}$ & $3 \cdot 5(17)$ & $14(92)$ & $4(5 \cdot 4)$ \\
\hline $\mathrm{Mn}^{(\mathrm{III})}(\mathrm{TDCPP}) \mathrm{OAc}$ & $1(7)$ & $3.6(37)$ & $3 \cdot 6(5 \cdot 3)$ \\
\hline $\mathrm{Mn}^{(\mathrm{III})}\left(\mathrm{TPNMe}_{2} \mathrm{TFPP}\right) \mathrm{OAc}$ & $3(20)$ & $8(70)$ & $2 \cdot 6(3 \cdot 5)$ \\
\hline
\end{tabular}

${ }^{\text {a }}$ Reactions were performed under air at room temperature with a catalyst:imidazole: tetralin:cyclooctene: oxidant : PTC molar ratio of $1: 10: 250: 83: 167: 10$ as described in the experimental after $0 \cdot 5 \mathrm{~h}(8 \mathrm{~h})$. Repeated experiments confirmed $>95 \%$ accuracy; ${ }^{b}$ Yields $\%$ refer to isolated yields of products; ${ }^{\mathrm{c}}(\mathrm{B}) /(\mathrm{A})$ corresponds to the ratio of cyclooctene oxide/á-tetralone yields. Imidazole could not be oxidized under these reaction conditions

Table 2. Competitive oxygenation of tetralin and cyclooctene with sodium periodate catalyzed by different manganese porphyrins in the absence of imidazole. ${ }^{\mathrm{a}}$

\begin{tabular}{lccc}
\hline Catalyst & Yield \% of $\alpha$-tetralone $(\mathrm{A})^{\mathrm{b}}$ & ${\text { Yield } \% \text { of cyclooctene oxide }(\mathrm{B})^{\mathrm{b}}}^{(\mathrm{B}) /(\mathrm{A})^{\mathrm{c}}}$ \\
\hline $\mathrm{Mn}^{(\mathrm{III})}$ (TPP)OAc & $7(17)$ & $34(79)$ & $4 \cdot 8(4 \cdot 6)$ \\
$\mathrm{Mn}^{(\mathrm{IIII})}$ (TNP)OAc & $4 \cdot 5(16)$ & $23(87)$ & $5 \cdot 1(5 \cdot 4)$ \\
$\mathrm{Mn}^{(\mathrm{III})}$ (TMP)OAc & $3(10 \cdot 5)$ & $28(86)$ & $9.3(8 \cdot 2)$ \\
$\mathrm{Mn}^{(\mathrm{IIII})}$ (TDCPP)OAc & $2(4 \cdot 5)$ & $3(15)$ & $1 \cdot 5(3 \cdot 3)$ \\
$\mathrm{Mn}^{(\mathrm{III})}$ (TPNMe ${ }_{2}$ TFPP)OAc & $2 \cdot 5(6)$ & $2(19)$ & $0 \cdot 8(3 \cdot 1)$ \\
\hline
\end{tabular}

${ }^{\mathrm{a}}$ Reactions were performed under the same conditions as described in table 1; ${ }^{\mathrm{b}}$ Progress of the reactions was analyzed by GLC after $1 \mathrm{~h}(8 \mathrm{~h}) ;{ }^{\mathrm{c}}(\mathrm{B}) /(\mathrm{A})$ refers to the ratio of cyclooctene oxide/ $\alpha$-tetralone yields

their stability toward oxidative breakdown and eventually led to high catalytic activity. ${ }^{18,22}$

Electron-withdrawing aryl-substituents on the periphery of the porphyrin ligand declined the catalytic activity of the oxygenation system. (Im) $\mathrm{Mn}^{\text {(III) }}$ (TDCPP)OAc and (Im) $\mathrm{Mn}^{(\mathrm{III})}$ (TPNMe ${ }_{2}$ TFPP)OAc resulted in $0.18 \mathrm{mmol}$ and $0.10 \mathrm{mmol}$ of epoxide and $\alpha$-tetralone respectively, after $8 \mathrm{~h}$, whereas clearly higher conversions were obtained with $(\operatorname{Im}) \mathrm{Mn}^{(\mathrm{III})}$ (TPP)OAc, (Im) $\mathrm{Mn}^{\text {(III) }}$ (TNP)OAc, and (Im) $\mathrm{Mn}^{\text {(III) }}$ (TMP)OAc catalysts under the same reaction conditions. They provided $0.64,0.68$, and $0.715 \mathrm{mmol}$ of the oxygenated products after $8 \mathrm{~h}$ respectively. Our findings revealed that the ratio of cyclooctene oxide/ $\alpha$ tetralone extended between 2.6 and 3.2 for sterically unhindered (Im) $\mathrm{Mn}^{\text {(III) }}$ (TPP)OAc, (Im) $\mathrm{Mn}^{\text {(III) }}$ (TNP) $\mathrm{OAc}$, and (Im) Mn ${ }^{\text {(III) }}$ (TPNMe ${ }_{2}$ TFPP)OAc catalysts in short reaction times. This ratio varies between 3.6 and 4 for $(\mathrm{Im}) \mathrm{Mn}^{\text {(III) }}$ (TMP)OAc and (Im) $\mathrm{Mn}^{\text {(III) }}$ (TDCPP)OAc, resulting from the steric hindrances induced by mesityl- and 2,6-dichlorophenyl-groups of the meso-aryl rings.

Table 2 presents the results of competitive oxygenation of tetralin and cyclooctene in the absence of imidazole. Comparison of these findings with those provided in table 1 , reveals that $\mathrm{Mn}^{\text {(III) }}$ (TDCPP)OAc and $\mathrm{Mn}^{\text {(III) }}$ (TPNMe ${ }_{2}$ TFPP)OAc are more reactive in the oxygenation of tetralin than in the epoxidation of cyclooctene, in the absence of imidazole. The ratio of cyclooctene oxide/ $\alpha$-tetralone changed between 0.8 to 3.3 for these two catalysts in the absence of imidazole.

In the absence of imidazole, the sterically unhindered catalysts, $\mathrm{Mn}^{\text {(III) }}$ (TPP)OAc and $\mathrm{Mn}^{(\mathrm{III})}(\mathrm{TNP}) \mathrm{OAc}$, were 4.6-5.4 times more efficient in the epoxidation of cyclooctene than in the oxygenation of tetralin after $8 \mathrm{~h}$. It seems that coordination of imidazole to the metal center decreased the activity of $\mathrm{Mn}^{\text {(III) }}$ (TPP)OAc and $\mathrm{Mn}^{\text {(III) }}$ (TNP)OAc toward epoxidation of cyclooctene. For a sterically demanding catalyst, $\mathrm{Mn}^{(\mathrm{III})}$ (TMP)OAc, the ratio of cyclooctene oxide/ $\alpha$ tetralone approached 8.0-9.0 in the absence of axial ligand.

Higher-valent oxo-manganese porphyrin intermediates have been characterized and are generally proposed as reactive oxidizing species in the oxygenation systems catalyzed by metalloporphyrins. ${ }^{14,15,23,24}$ It is believed that imidazole strongly affects the stability and reactivity of these intermediates in the oxygenation reactions. ${ }^{25-27}$ Findings have shown that these 


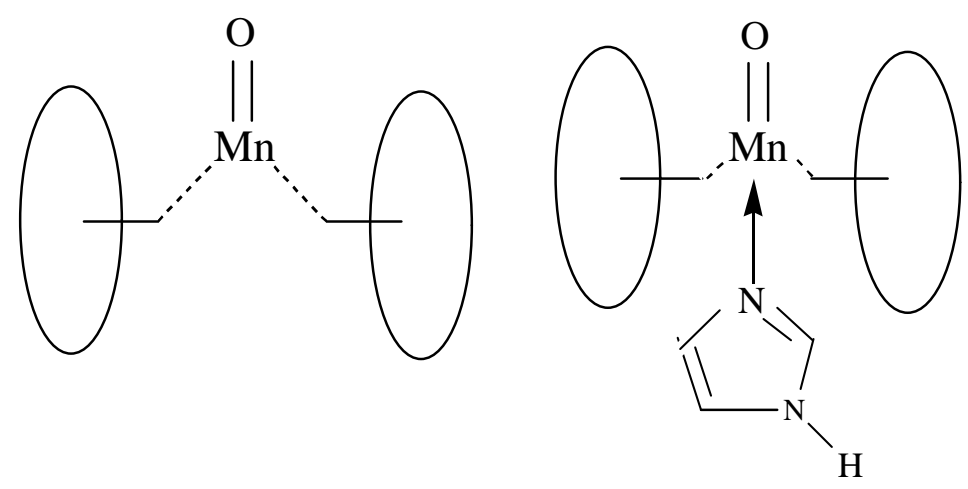

Figure 1. Effect of imidazole on the displacement of Mn from the mean plane of the porphyrin toward the oxo ligand.

active oxidizing species affect product selectivity, stereoselectivity, and regioselectivity of oxidations catalyzed by Mn porphyrins. ${ }^{28,29}$

Our preliminary ultraviolet studies have confirmed generation of a high-valent oxidizing species by observation of a Soret band at about $420 \mathrm{~nm}$ in sodium periodate-manganese(III) porphyrin oxygenation system. The manganese atom in the proposed oxo-manganese species should bear (IV) or (V) oxidation state, depending on the mechanism of oxidation reactions. To the best of our knowledge, formation of the oxo-manganese $(\mathrm{V})$ is more reliable for $\mathrm{NaIO}_{4}$ Mn(Por) oxidation system.

The results of tables 1 and 2 allow us to modulate the observed substrate selectivity in the presence or absence of imidazole as Mn axial ligand. This imidazole effect could be interpreted if we admit that the active oxygen complex is a hexacoordinated $\mathrm{Mn}^{(\mathrm{V})}$ oxo complex with an imidazole ligand in transposition with respect to the oxo ligand (figure 1). Presumably, binding of imidazole to the Mn-center would restrict the displacement of Mn atom from the mean plane of the spacious porphyrin toward the oxo ligand and thus limit the access of more spacious substrate, tetralin, to the $\mathrm{Mn}^{(\mathrm{V})}=\mathrm{O}$ entity. ${ }^{30}$

\section{Conclusion}

This study showed that porphyrin structure, and electronic, and intrinsic reactivity of the active oxidizing oxo species, ${ }^{14,15}$ have a marked influence on the ratio of cyclooctene oxide to $\alpha$-tetralone in competitive oxidation of cyclooctene and tetralin with $\mathrm{NaIO}_{4}$. According to the above findings, selectivity for cyclooctene epoxidation, in comparison with tetralin oxygenation, was decreased in the presence of axial ligand for TPP, TNP, and TMP porphyrins; whereas a pronounced increase was observed for the electron-deficient porphyrin ligands, TDC and TPFPP, in the presence of imidazole.

In conclusion, our findings $\mathrm{s}^{13,20}$ have proved that axial ligation of nitrogen donors to the manganese atom slightly affect the selectivity of oxidation reactions and rate of oxygenation decreases in the presence of axial ligand. As described above, this is in direct contrast to other reports. Further investigations will be necessary to determine different roles played by nitrogen donors in this oxidation system compared with other similar oxygenation routes catalyzed by manganese porphyrins.

\section{Acknowledgements}

I appreciate the financial support by the Sabzevar Teacher Education University. My grateful thanks also extend to Dr D Mohajer for his useful suggestions.

\section{References}

1. Mansuy D 1993 Coord. Chem. Rev. 125129

2. Marques M H and Brown K L 2002 Coord. Chem. Rev. 225123

3. Fermi G and Perutz M F 1981 Hemoglobin and myoglobin. Atlas of biological structures (eds) D C Philips and M Richards (Oxford: Clarendon)

4. Meunier B 1992 Chem. Rev. 921411

5. Groves G T 2000 J. Porphyrins Phthalocyanins 4350

6. Mansuy D, Battioni P and Renaud J P 1984 J. Chem. Soc., Chem. Commun. 1255

7. Srinivasan K, Michaud P and Kochi J K 1986 J. Am. Chem. Soc. 1082309

8. Momenteau M and Reed C A 1994 Chem. Rev. 94 659 
9. Battioni P, Renaud J P, Bartoli J F, Reina-Artiles M, Fort M and Mansuy D $1988 \mathrm{~J}$. Am. Chem. Soc. 110 8462

10. Montanari F, Banfi S and Quici S 1989 Pure Appl. Chem. 611631

11. Kadish K M and Kelly S 1979 Inorg. Chem. 182968

12. Kelly S and Kadish K M 1982 Inorg. Chem. 213631

13. Mohajer D and Tayebee R 1999 Iranian J. Sci. Tech. 1827

14. Mohajer D, Tayebee R and Goudarziafshar H $1999 \mathrm{~J}$. Chem. Res. (S) 168

15. Mohajer D, Tayebee R and Goudarziafshar H $1998 J$. Chem. Res. (S) 822

16. Mansuy D, Renaud J P, Battioni P and Bartoli J T 1985 J. Chem. Soc., Chem. Commun. 888

17. Meunier B, Guilmet E, De Carvalho M and Poilblanc R 1984 J. Am. Chem. Soc. 1066668

18. Lindsey S and Wagner R W 1989 J. Org. Chem. 54 828

19. Adler A D, Longo F R, Finarelli J D, Goldmacher G, Assour J and Korsakoff L 1967 J. Org. Chem. 32476

20. Tayebee R 2002 Indian J. Chem. B41 2190
21. Mohajer D, Banihashemi A and Nasiri B 1996 Iranian J. Sci. Tec. 1827

22. Mansuy D, Bartoli J F and Momenteau M 1982 Tetrahedron Lett. 232781

23. Kin-Botulinski M and Meunier B 1988 Inorg. Chem. 27209

24. Groves J T, Watanabe Y and Mc Murry T J 1983 J. Am. Chem. Soc. 1054489

25. Traylor T G, Lee W A and Stynes D V J 1984 J. Am. Chem. Soc. 106755

26. Yuan L C and Bruice T C 1986 J. Am. Chem. Soc. 1081643

27. Lee R W, Nakagaki P C and Bruice T C 1989 J. Am. Chem. Soc. 1111368

28. Suslick K S and Cook B R 1987 J. Chem. Soc., Chem. Commun. 200

29. Groves J T and Nemo T E 1983 J. Am. Chem. Soc. 1055786

30. Penner-Hahn J E, Mc Murry T G, Renner M, LatosGrazynsky L, Ebel K S, Davis I M, Balch A L, Groves J T, Dawson J H and Hodgson K O 1983 J. Bio. Chem. 25812761 